\title{
Sensitivity Invariants for Nonlinear Networks
}

\author{
M. N. S. SWAMY, CHAMPA BHUSHAN, AND K. THULASIRAMAN
}

\begin{abstract}
For a general class of nonlinear networks, explicit expressions for sensitivities of a response due to nonlinear elements are derived. These expressions are used to establish invariance relationships for the sums of sensitivities over different sets of parameters of two classes of nonlinear networks. It is indicated how these relationships can be used to establish the invariant nature of the sum of higher order sensitivities and a lower bound for the quadratic sensitivity index.
\end{abstract}

\section{INTRODUCTION}

$\mathrm{T}$ HE PROPERTY of sensitivity invariance in linear time-invariant networks has generated much interest in the literature. For a number of classes of passive and active lumped networks, several authors have shown the existence of such invariants [1]-[7]. Recently, Sablatash and Seviora [8] established, using the concept of adjoint networks [9], sensitivity invariants for a class of active networks containing uniform transmission lines. Subsequently, the authors extended these results to networks containing nonuniform transmission lines and voltage-to-current and current-to-voltage transducers simultaneously [10]. The use of these invariants in optimal synthesis (optimal in the sense that the multiparameter sensitivity performance has been minimized) has been considered in [11], while its use in establishing lower bounds for the quadratic sensitivity index has been discussed in [6], [10], and [12]. A good survey, along with a number.of references on the subject of sensitivity, has appeared recently [13].

No such invariant relations have been obtained for nonlinear networks. With the increasing use of nonlinear elements in circuit design [14], such invariant relationships for nonlincar networks should be useful in computer-aided circuit design. In this paper we establish sensitivity invariants for nonlinear networks, and these offer a promising means of determining lower bounds for quadratic sensitivity index. In Section II we establish formulas for sensitivities with respect to various parameters. In Section III sensitivity-invariant relationships are established for different combinations of responses and excitations. Sensitivities of response

Manuscript received March 5, 1972; revised July 17, 1972. This paper is based on part of a D.Eng. dissertation submitted by $C$. Bhushan, under the supervision of M. N. S. Swamy, to Sir George Williams University, Montreal, Que., Canlada, and was supported by the National Research Council of Canada under Grant A-7739.

M. N. S. Swamy and C. Bhushan are with the Department of Electrical Engineering, Sir George Williams University, Montreal, Que., Canada:

$\mathrm{K}$. Thulasiraman is with the Department of Electrical Engineering, Sir Gcorge Williams University, Montreal, Que., Canada, on leave from the Department of Electrical Engineering, Indian Institute of Technology, Madras, India. due to changes in excitation amplitudes are also taken into consideration in establishing the invariance relationships.

\section{Sensitivity Components Due to Nonlinear Elements}

In this section we will calculate the sensitivities of a response due to nonlinear elements.

Consider a general linear/nonlinear time-invariant network consisting of current-controlled and voltagecontrolled resistors (CCR and VCR), voltage-controlled and charge-controlled capacitors (VCC and QCC), current-controlled and flux-controlled inductors (CCL and FCL) [14], current-to-current transducers (CCT), current-to-voltage transducers (CVT's), voltage-tocurrent transducers (VCT), voltage-to-voltage transducers (VVT), voltage- and current-controlled gyrators (VCG and CCG), hybrid gyrators (HG), and impedance converters (IC). Let us denote such a network by $N$ and its adjoint by $N^{A}$.

We will use $N$ and $N^{A}$ and follow the procedure of Seth and Singhal [15] to obtain explicit formulas for sensitivities due to the above nonlinear elements. Let the network $N$ be considered at time $\xi$ and $N^{A}$ at time $\tau=\ell-\xi$. We know by Tellegen's theorem that

$$
\begin{aligned}
\sum_{p} \Delta v_{p}(\xi) \phi_{p}(t & -\xi)-\Delta i_{p}(\xi) \psi_{p}(t-\xi) \\
& =\sum_{e} \Delta v_{e}(\xi) \phi_{e}(t-\xi)-\Delta i_{e}(\xi) \psi_{e}(t-\xi)
\end{aligned}
$$

where $i_{p}\left(\phi_{p}\right)$ and $v_{p}\left(\psi_{p}\right)$ denote currents through and voltages across the ports of $N\left(N^{A}\right), i_{e}\left(\phi_{e}\right)$ and $v_{e}\left(\psi_{e}\right)$ are the currents through and voltages across internal elements, and $\Delta v_{p}, \Delta i_{p}, \Delta v_{e}$, and $\Delta i_{e}$ are, respectively, the changes in $v_{p}, i_{p}, v_{e}$, and $i_{e}$ due to a small change in the parameters of network $N$.

Integrating (1) from 0 to $t$, we get

$$
\begin{aligned}
& \sum_{p} \int_{0}^{t}\left[\Delta v_{p}(\xi) \phi_{p}(t-\xi)-\Delta i_{p}(\xi) \psi_{p}(t-\xi)\right] d \xi \\
& \quad=\sum_{e} \int_{0}^{t}\left[\Delta v_{e}(\xi) \phi_{e}(t-\xi)-\Delta i_{e}(\xi) \psi_{e}(t-\xi)\right] d \xi
\end{aligned}
$$

Denoting

$$
x * y=\int_{0}^{\imath} x(\xi) y(t-\xi) d \xi
$$

we may rewrite (2a) in the form

$$
\sum_{p}\left(\Delta v_{p} * \phi_{p}-\Delta i_{p} * \psi_{p}\right)=\sum_{e}\left(\Delta v_{e} * \phi_{e}-\Delta i_{e} * \psi_{e}\right) .
$$


Let us first consider a VCR branch described by

$$
i_{G}=a_{G} f_{v G}\left(\beta_{G} v_{G}\right)
$$

where $f_{r} G$ is assumed to be a single-valued function of $v_{G} \cdot{ }^{1}$

For small variations in the parameters $a_{G}$ and $\beta_{G}$, we have

$$
\Delta i_{G}=\Delta a_{G} f_{v G}\left(\beta_{G v_{G}}\right)+a_{G} f_{v G}{ }^{\prime}\left(\beta_{G} v_{G}\right)\left[\beta_{G} \Delta v_{G}+v_{G} \Delta \beta_{G}\right]
$$

where the prime denotes differentiation with respect to $\left(\beta_{G} v_{G}\right)$, which is assumed to exist. The contribution to the right-hand side of (2) due to the terms corresponding to this element is hence given by

$$
\begin{aligned}
\int_{0}^{t} \Delta v_{G}(\xi) & {\left[\phi_{G}(t-\xi)-a_{G} \beta_{G} f_{v G^{\prime}}\left(\beta_{G} v_{G}(\xi)\right) \psi_{G}(t-\xi)\right] d \xi } \\
& -\frac{\Delta a_{G}}{a_{G}}\left(i_{G} * \psi_{G}\right) \\
& -\Delta \beta_{G} \int_{0}^{t} a_{G} f_{v G^{\prime}}\left(\beta_{G} v_{G}(\xi)\right) v_{G}(\xi) \psi_{G}(t-\xi) d \xi .
\end{aligned}
$$

If the corresponding branch in $N^{A}$ is defined by

$$
\phi_{G}(t-\xi)=a_{G} \beta_{G} f_{v} G^{\prime}\left\{\beta_{G^{v}}(\xi)\right\} \psi_{G}(t-\xi)
$$

then (4) reduces to

$$
-\frac{\Delta a_{G}}{a_{G}}\left(i_{G} * \psi_{G}\right)-\frac{\Delta \beta_{G}}{\beta_{G}}\left(v_{G} * \phi_{G}\right) .
$$

The first term in (6) gives the sensitivity component due to $a_{G}$, while the second term gives that due to $\beta_{G}$.

Similarly, for a CCR described by

$$
v_{R}=\alpha_{R} f_{i R}\left(b_{R} i_{R}\right)
$$

it may be shown that the sensitivity components due to $\alpha_{R}$ and $b_{R}$ are, respectively,

$$
\frac{\Delta \alpha_{R}}{\alpha_{R}}\left(v_{R} * \phi_{R}\right)
$$

and

$$
\frac{\Delta b_{R}}{b_{R}}\left(i_{R} * \psi_{R}\right)
$$

by choosing the adjoint element to be

$$
\psi_{R}(t-\xi)=\alpha_{R} b_{R} f_{i R^{\prime}}\left\{b_{R} i_{R}(\xi)\right\} \phi_{R}(t-\xi) .
$$

If the resistor happens to be linear,

$$
v_{R}=R i_{R} \quad \text { or } \quad i_{G}=G v_{G}
$$

we may associate $G$ with either $a_{G}$ or $\beta_{G}$, or $R$ with $b_{G}$ or $\alpha_{G}$.

\footnotetext{
1 For example, in the case of a junction diode governed by $i=I_{s}\left[\exp \left(q^{v} / K T\right)-1\right], I_{s}$ being the reverse saturation constant, $q$ and $K$ being constants, and $T$ the temperature, we may identify $I_{s}$ and $q /(K T)$ with $a$ and $\beta$, respectively, in (3). A variation in $I_{s}$ corresponds to a variation in the parameter $a$, while a change in $T$ would cause a change in $\beta$.
}

Let us next consider a VCC branch described by

$$
q_{C}=a_{C} f_{v C}\left(\beta_{C^{v}}\right) \quad i_{C}=\frac{d}{d \xi}\left(q_{C}\right) .
$$

A small variation in parameters gives

$$
\begin{aligned}
& \Delta i_{C}=\frac{d}{d \xi}\left[\Delta a_{C} f_{v C}\left(\beta_{C} v_{C}\right)+a_{C} f_{v C^{\prime}}\left(\beta_{C} v_{C}\right) \beta_{C} \Delta v_{C}\right. \\
& \left.+a_{C} f_{v C^{\prime}}\left(\beta_{C} v_{C}\right) v_{C} \Delta \beta_{C}\right]
\end{aligned}
$$

where the prime denotes differentiation with respect to $\left(\beta_{C} v_{C}\right)$. The contribution to the right-hand side of (2) due to the terms corresponding to this element is given by

$$
\begin{aligned}
& \int_{0}^{t}\left\{\Delta v_{C}(\xi) \phi_{C}(t-\xi)-\frac{d}{d \xi}\left[\Delta a_{C} f_{v C}\left(\beta_{C} v_{C}\right)+a_{C} f_{v C^{\prime}}\left(\beta_{C} v_{C}\right) \beta_{C} \Delta v_{C}\right.\right. \\
& \left.\left.+a_{C} f_{v C^{\prime}}\left(\beta_{C} v_{C}\right) v_{C} \Delta \beta_{C}\right] \psi_{C}(t-\xi)\right\} d \xi \\
& =\int_{0}^{t}\left\{\Delta v_{C}(\xi)\left[\phi_{C}(t-\xi)+a_{C} \beta_{C} f_{v} C^{\prime}\left(\beta_{C} v_{C}\right) \frac{d}{d \xi} \psi_{C}(t-\xi)\right]\right\} d \xi \\
& -\left[a_{C} \beta_{C} f_{v C}\left(\beta_{C^{v}}\right) \Delta v_{C} \psi_{C}(t-\xi)\right]_{0}{ }^{t}-\frac{\Delta a_{C}}{a_{C}}\left(i_{C} * \psi_{C}\right) \\
& -\left[a_{C} f_{v C^{\prime}}\left(\beta_{C} v_{C}\right) v_{C} \Delta \beta_{C} \psi_{C}(t-\xi)\right]_{0}^{t} \\
& +\int_{0}^{t} a_{C} f_{v} C^{\prime}\left(\beta_{C} v_{C}\right) v_{C} \Delta \beta_{C} \frac{d}{d \xi} \psi_{C}(t-\xi) d \xi \text {. }
\end{aligned}
$$

If the corresponding branch in $N^{A}$ is defined by

$$
\phi_{C}(\tau)=a_{C} \beta_{C} f_{v C^{\prime}}\left(\beta_{C^{v} C}\right) \frac{d}{d \tau} \psi_{C}(\tau), \quad \tau=(t-\xi)
$$

the first term on the right-hand side of (12) is zero. The second term on the right-hand side of (12) is

$$
a_{C} \beta_{C}\left[f_{v C^{\prime}}\left(\beta_{C} v_{C}(0)\right) \Delta v_{C}(0) \psi_{C}(t)-f_{v C}{ }^{\prime}\left(\beta_{C} v_{C}(t)\right) \Delta v_{C}(t) \psi_{C}(0)\right] .
$$

The second term in the above equation is set to zero by choosing the initial condition $\psi_{C}(0)=0$ in $N^{A}$. The first term is either equal to zero for a given initial condition in $N$, or gives the sensitivity component with respect to the initial condition.

The third term on the right-hand side of (12) gives the sensitivity component with respect to parameter $a_{C}$. The fourth and fifth terms on the right-hand side of (12) can be written by using (13) and by noting that $\psi_{C}(0)=0$ as

$$
-\frac{\Delta \beta_{C}}{\beta_{C}}\left[-a_{C} \beta_{C} f_{C v}{ }^{\prime}\left(\beta_{C^{v} C}(0)\right) v_{C}(0) \psi_{C}(t)+\left(v_{C} * \phi_{C}\right)\right]
$$

which gives the sensitivity component due to $\beta_{C}$.

Let us next consider a nonlinear VVT described as in Table I. A variation in the parameters $\alpha_{V V}$ and $\beta_{V V}$ gives $\Delta v_{2}=\Delta \alpha_{V V} f_{V V}\left(\beta_{V V} v_{1}\right)+\alpha_{V V} f_{V V}^{\prime}\left(\beta_{V V} v_{1}\right) \beta_{V V} \Delta v_{1}$

$$
+\alpha_{V V} f_{V V}^{\prime}\left(\beta_{V V} v_{1}\right) v_{1} \Delta \beta_{V V}
$$


and

$$
\Delta i_{1}=0
$$

where the prime denotes differentiation with respect to $\left(\beta_{v v v_{1}}\right)$. The contribution to the right-hand side of (2) due to the terms corresponding to this element is given by

$$
\begin{aligned}
& \int_{0}^{t}\left\{\Delta v_{1}\left[\phi_{1}(t-\xi)+\alpha_{V V} \beta_{V V} f_{V V}{ }^{\prime}\left(\beta_{V V} v_{1}(\xi)\right) \phi_{2}(t-\xi)\right]\right. \\
& \left.-\Delta i_{2}(\xi) \psi_{2}(t-\xi)\right\} d \xi+\frac{\Delta \alpha_{V V}}{\alpha_{V V}}\left(v_{2} * \phi_{2}\right) \\
& \quad+\frac{\Delta \beta_{V V}}{\beta_{V V}} \int_{0}^{t} \alpha_{V V} \beta_{V V} f_{V V}\left(\beta_{V V} v_{1}(\xi)\right) v_{1}(\xi) \phi_{2}(t-\xi) d \xi
\end{aligned}
$$

If the corresponding element in $N^{A}$ is given by

$$
\begin{aligned}
& \phi_{1}(t-\xi)=-\alpha_{V V} \beta_{V V} f_{V V}^{\prime}\left(\beta_{V V} v_{1}(\xi)\right) \phi_{2}(t-\xi) \\
& \psi_{2}(t-\xi)=0
\end{aligned}
$$

then the first term in (15) is zero, and the second term gives the sensitivity component with respect to $\alpha_{V V}$. The last term in (15) can be written with the help of (16) as

$$
-\frac{\Delta \beta_{V V}}{\beta_{V V}}\left(v_{1} * \phi_{1}\right)
$$

which gives the sensitivity component due to $\beta_{V V}$.

In a similar manner, the adjoint descriptions and the sensitivity components due to all the other elements can be found out, and they are listed in Table I. It is to be noted that all the different functions $f$ are assumed to be single valued and differentiable. It should also be pointed out (Table I) that the parameter symbols $a$ and $b$ are always associated with the current variables, while $\alpha$ and $\beta$ are associated with the voltage variables; further, the symbol $b$ is always associated with an independent current variable, while the symbol $\beta$ is associated with an independent voltage variable. These symbols have been chosen in this manner so that the invariant relationships to be derived in Section III may be written in a compact way.

\section{Sensitivity Invariants for Nonlinear Networks}

Consider the general $n$-port network $N$ defined in the previous section. Let $N^{A}$ be its adjoint. Let the network $N$ be considered at time $\xi$ and $N^{A}$ at time $\tau=t-\xi$. We know by Tellegen's theorem that

$$
\sum_{p} i_{p}(\xi) \psi_{p}(t-\xi)=\sum_{e} i_{e}(\xi) \psi_{e}(t-\xi)
$$

and

$$
\sum_{p} v_{p}(\xi) \phi_{p}(t-\xi)=\sum_{e} v_{e}(\xi) \phi_{e}(t-\xi)
$$

where $i_{e}\left(\psi_{e}\right)$ and $i_{p}\left(\psi_{p}\right)$ are, respectively, the currents through (voltages across) interior elements and the external ports for the original (adjoint) network, while $v_{e}\left(\phi_{e}\right)$ and $v_{p}\left(\phi_{p}\right)$ are, respectively, the voltage across (current through) interior elements and external ports for the original (adjoint) network.

Integrating (18) and (19) from 0 to $t$, we get

$$
\sum_{p}\left(i_{p} * \psi_{p}\right)=\sum_{e}\left(i_{e} * \psi_{e}\right)
$$

and

$$
\sum_{p}\left(v_{p} * \phi_{p}\right)=\sum_{e}\left(v_{e} * \phi_{e}\right)
$$

Let the sensitivity of any response $U$ with respect to a parameter $p_{k}$ be defined as

$$
S_{p_{k}} U=p_{k} \frac{\partial U}{\partial p_{k}} .
$$

We shall now establish some sensitivity-invariant relations for two classes of nonlinear networks with different types of excitations and responses. We shall denote by $N_{1}$ that class of nonlinear networks $N$ where either the QCC's and CCL's are absent or, if present, their initial conditions $q_{C}(0)=i_{L}(0)=0$. Also, we shall denote by $N_{2}$ that class of nonlinear networks $N$ where either the VCC's and the FCL's are absent or, if present, their initial conditions $v_{C}(0)=\eta_{L}(0)=0$. We will first obtain sensitivity-invariant relations with different types of excitations and responses, assuming changes only in the parameters $a, b, \alpha$, and $\beta$ in the different network elements.

\section{Case 1: Voltage Response Due to Voltage Excitation}

Consider the network $N_{1}$ whose adjoint is denoted by $N_{1}{ }^{A}$. Let $v_{2}$ denote the open-circuit voltage response at port 2 of $N_{1}$ due to a voltage excitation at port 1 , all other ports being open. Let port 2 of $N_{1}{ }^{A}$ be excited by an impulse current function $\delta(\tau)$, with port 1 shorted and all other ports open circuited. Then the left-hand side of (2) can be written as

$$
\int_{0}^{t} \Delta v_{2}(\xi) \delta(t-\xi) d \xi=\Delta v_{2}(t) .
$$

The right-hand side of (2) gives the contributions to this change $\Delta v_{2}(t)$ due to the variations in the different parameters $a^{\prime} \mathbf{s}, \alpha$ 's, $b^{\prime}$ s, and $\beta^{\prime}$ 's of the network elements in $N_{1}$. These have already been tabulated in Table I. For example, the contribution due to the $a_{G}$ of a VCR is given by

$$
-\frac{\Delta a_{G}}{a_{G}}\left(i_{G} * \psi_{G}\right) .
$$

Thus from (23) and (24) we have

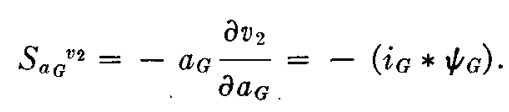


TABLE I

\begin{tabular}{|c|c|c|c|c|}
\hline $\begin{array}{c}\text { Element } \\
\text { Classification }\end{array}$ & $\begin{array}{l}\text { Description } \\
\text { of } \\
\text { the Element }\end{array}$ & $\begin{array}{l}\text { Adjoint } \\
\text { Description for Senoitivity } \\
\text { Computation at time } t(\tau=t-\xi)\end{array}$ & $\begin{array}{l}\text { Contribution to the } \\
\text { due to a change it } \\
\text { a or b }\end{array}$ & $\begin{array}{c}\text { fight-hand stde of (2) } \\
\text { the parameter } \\
\alpha \text { or } \beta \\
\end{array}$ \\
\hline \begin{tabular}{|c} 
Voltage-controlled \\
resistance \\
(VCR)
\end{tabular} & $I_{G}=a_{G}{ }^{f} v_{G}\left(B_{G} v_{G}\right)$ & $\phi_{G}(\tau)={ }_{G} \beta_{G}{ }^{f}{ }_{v G}\left(\beta_{G}{ }_{G}(\xi)\right) \psi_{G}(\tau)$ & $-\frac{\Delta a_{G}}{a_{G}}\left(i_{G} * \Psi_{G}\right)$ & $-\frac{\Delta B_{G}}{B_{G}}\left(v_{G} * \phi_{G}\right)$ \\
\hline $\begin{array}{c}\text { Current-controlled } \\
\text { resistance } \\
\text { (CCR) }\end{array}$ & $v_{R}=\alpha_{R} f_{1 R}\left(b_{R} 1_{R}\right)$ & $\Psi_{R}(\tau)=a_{R} b_{R}^{f}{ }_{I R}^{\prime}\left(b_{R}{ }^{1}(\xi)\right) \phi_{R}(\tau)$ & $\frac{\Delta \mathrm{b}_{\mathrm{R}}}{\mathrm{b}_{\mathrm{R}}}\left(1_{\mathrm{R}} * \Psi_{\mathrm{R}}\right)$ & $\frac{\Delta \alpha_{R}}{\alpha_{R}}\left(v_{R} * \phi_{R}\right)$ \\
\hline $\begin{array}{l}\text { Voltage-controlled } \\
\text { capacitance } \\
\text { (VCC) }\end{array}$ & $\begin{array}{c}q_{C}=a_{C} f_{v C}\left(\beta_{C} v_{C}\right) \\
i_{c}=\frac{d}{d \xi} q_{C}\end{array}$ & $\begin{array}{c}\Phi_{C}(\tau)={ } c^{\beta} c^{f}{ }_{v C}^{\prime}\left(\beta_{C}{ }^{v} c(\xi)\right) \\
\times \frac{d}{d \tau} \psi_{C}(\tau)\end{array}$ & $-\frac{\Delta a_{c}}{a_{c}}\left(i_{c} * \psi_{c}\right)$ & $\begin{array}{l}-\frac{\Delta{ }^{B} c}{{ }^{B} c}\left[v_{c} * \phi_{C}-\right. \\
{ }^{-B} c^{a} c^{v} c^{(0) \psi_{c}(t)} \\
\left.\times f_{v c}^{\prime}\left(\beta_{c} v_{c}(0)\right)\right]\end{array}$ \\
\hline $\begin{array}{l}\text { Charge-controlled } \\
\text { capac1tance } \\
\text { (QCC) }\end{array}$ & $\begin{array}{l}\left.v_{C}=\alpha_{C}{ }^{f} c^{(b} c^{q_{C}}\right) \\
1_{C}=\frac{d}{d \xi} q_{C}\end{array}$ & 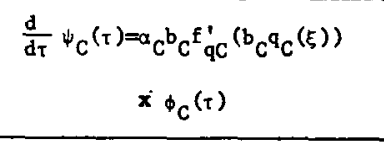 & $\begin{array}{r}\frac{{ }^{\Delta b} \mathrm{c}}{{ }^{b_{c}}\left[1_{c}\right.}{ }^{*} \psi_{c} \\
\left.+q_{c}(0) \psi_{c}(t)\right]\end{array}$ & $\frac{{ }^{\Delta \alpha_{c}}}{{ }^{\alpha_{c}}}\left(v_{c} * \phi_{c}\right)$ \\
\hline $\begin{array}{l}\text { Flux-controlled } \\
\text { Inductor } \\
\text { (FCL) }\end{array}$ & $\begin{array}{c}f_{L}=a_{L} f_{n L}\left(\beta_{L} \eta_{L}\right) \\
v_{L}=\frac{d \eta_{L}}{d \xi}\end{array}$ & $\begin{aligned} \frac{d}{d \tau} \phi_{L}(\tau)= & a_{L} \beta_{L} f_{n L}^{\prime}\left(\beta_{L} \eta_{L}(\xi)\right) \\
& \times \psi_{L}(\tau)\end{aligned}$ & $-\frac{\Delta a_{L}}{a_{L}}\left(1_{L} * \psi_{L}\right)$ & $\begin{array}{l}-\frac{\Delta \beta_{L}}{\beta_{L}}\left[v_{L} * \phi_{L}\right. \\
\left.+\eta_{L}(0) \phi_{L}(t)\right]\end{array}$ \\
\hline $\begin{array}{l}\text { Current-controlled } \\
\text { Inductor } \\
\text { (CCL) }\end{array}$ & $\begin{array}{l}\eta_{L}=a_{L} f_{1 L}\left(b_{L} t_{L}\right) \\
v_{L}=\frac{d n_{L}}{d \xi}\end{array}$ & $\begin{aligned} \psi_{L}(\tau) & =\alpha_{L} b_{L} f_{i L}^{\prime}\left(b_{L} I_{L}(\xi)\right) \\
& \times \frac{d}{d \tau} \phi_{L}(\tau)\end{aligned}$ & $\begin{array}{l}\frac{\Delta b_{L}}{b_{L}}\left[1_{L} * \psi_{L}\right. \\
-a_{L} b_{L} 1_{L}(0) \phi_{L}(t) \\
\times f_{i L}^{\prime}\left(b_{L} 1_{L}(0)\right]\end{array}$ & $\frac{\Delta \alpha_{L}}{\alpha_{L}}\left(v_{L} * \phi_{L}\right)$ \\
\hline $\begin{array}{l}\text { Current-to-current } \\
\text { transducer } \\
\text { (CCT) }\end{array}$ & $\begin{array}{l}I_{2}=a_{c c} c^{f}\left(b c^{1}{ }_{1}\right) \\
v_{1}=0\end{array}$ & $\begin{array}{c}\left.\psi_{1}(\tau)=-a^{a} c^{b} c^{f^{\prime}} c c^{(b} c^{1} c_{1}(\varepsilon)\right) \\
\times \psi_{2}(\tau) \\
\phi_{2}(\tau)=0\end{array}$ & $\begin{array}{l}-\frac{\Delta \mathrm{a} c \mathrm{c}}{\mathrm{a}_{\mathrm{cC}}}\left(\mathrm{i}_{2} * \psi_{2}\right) \\
\frac{\Delta \mathrm{b} \mathrm{cc}}{\mathrm{b}_{\mathrm{cc}}}\left(\mathrm{f}_{1} * \psi_{1}\right)\end{array}$ & \\
\hline $\begin{array}{l}\text { Voltage-to-vol tage } \\
\text { transducer } \\
\text { (VVT) }\end{array}$ & $\begin{array}{c}\left.v_{2}=\alpha w^{f} w^{(\beta} w^{v_{1}}\right) \\
1_{1}=0\end{array}$ & $\begin{array}{c}\phi_{1}(\tau)=-\alpha v^{\beta} v^{f^{\prime}}\left(\beta_{w} v_{1}(\xi)\right) \\
\psi_{2}(\tau)=0 \quad \times \phi_{2}(\tau)\end{array}$ & & $\begin{array}{r}\frac{\Delta \alpha_{w}}{\alpha_{v w}}\left(v_{2} * \phi_{2}\right) \\
-\frac{\Delta \beta v v}{\beta_{v v}}\left(v_{1} * \phi_{1}\right)\end{array}$ \\
\hline $\begin{array}{l}\text { Voltage-to-current } \\
\text { transducer } \\
\text { (VCT) }\end{array}$ & $\begin{array}{c}1_{2}={ }^{=a} c^{f} v c^{\left(\beta_{v c} v_{1}\right)} \\
1_{1}=0\end{array}$ & $\begin{array}{c}\phi_{1}(\tau)=a_{v c^{\beta}} v c^{f^{\prime}}\left(\beta_{v c} v_{1}(\xi)\right) \\
\phi_{2}(\tau)=0 \quad x \psi_{2}(\tau)\end{array}$ & $-\frac{\Delta a_{v c}}{a_{v c}}\left(1_{2} * \psi_{2}\right)$ & $-\frac{\Delta \beta_{v c}}{\beta_{v c}}\left(v_{1} * \phi_{1}\right)$ \\
\hline $\begin{array}{l}\text { Current-to-vol tage } \\
\text { transducer } \\
\text { (CVT) }\end{array}$ & $\begin{array}{c}\left.v_{2}=\alpha c^{f} c v^{(b} c v^{1}\right) \\
v_{1}=0\end{array}$ & $\begin{array}{c}\psi_{1}(\tau)=\alpha \mathrm{CV}^{\mathrm{b}} \mathrm{cv} \mathrm{F}^{\prime}\left(\mathrm{b}_{\mathrm{CV}} \mathrm{v}_{1}(\xi)\right) \\
\quad \times \Phi_{2}(\tau) \\
\psi_{2}(\tau)=0\end{array}$ & $\frac{\Delta_{b} c v}{{ }^{b} c v}\left(1_{1} \star \psi_{2}\right)$ & $\frac{\Delta \alpha_{C V}}{a_{c V}}\left(v_{2} * \phi_{2}\right)$ \\
\hline $\begin{array}{l}\text { Voltage-controlled } \\
\text { gyrator } \\
\text { (VCG) }\end{array}$ & $\begin{array}{l}1_{1}=a_{V G 1 x} \\
f_{V G 1}\left(\bar{p}_{V G 1} v_{2}\right) \\
1_{2}=a_{v G 2} x \\
f_{V G 2}\left(B_{V G 2} v_{1}\right)\end{array}$ & 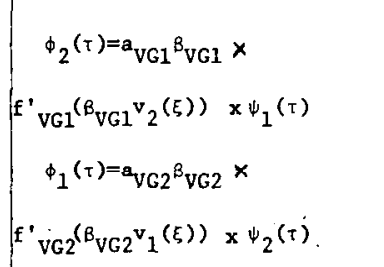 & $\begin{array}{l}-\frac{\Delta a_{v G 1}}{a_{\text {VG1 }}}\left(i_{1} * \psi_{1}\right) \\
-\frac{\Delta a_{\text {vG2 }}}{{ }^{2} v_{\text {G2 }}}\left(i_{2} * \psi_{2}\right)\end{array}$ & $\begin{array}{l}-\frac{\Delta B_{\mathrm{VG} 1}}{{ }_{\mathrm{VG} 1}}\left(\mathrm{v}_{2} * \phi_{2}\right) \\
-\frac{\Delta B_{\mathrm{VG} 2}}{B_{\mathrm{VG} 2}}\left(\mathrm{v}_{1} * \phi_{1}\right)\end{array}$ \\
\hline
\end{tabular}


TABLE I (Continued)

\begin{tabular}{|c|c|c|c|c|}
\hline $\begin{array}{l}\text { Current- } \\
\text { controlled } \\
\text { gyrator } \\
\text { (CCG) }\end{array}$ & $\begin{array}{l}v_{1}=a \operatorname{CGl}_{1} f_{C G 1}\left(b_{\left.\mathrm{CGI}_{2}{ }_{2}\right)}\right. \\
v_{2}=a_{\mathrm{CG} 2} \mathrm{f}_{\mathrm{CG} 2}\left(\mathrm{~b}_{\mathrm{CG} 2}{ }_{2}\right)\end{array}$ & 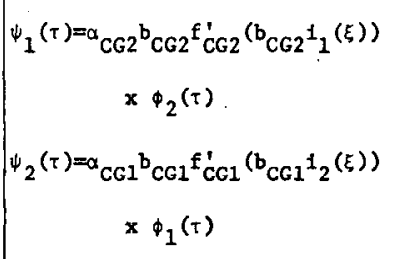 & $\begin{array}{l}\frac{\Delta b_{C G 1}}{b_{c G 1}}\left(i_{2} * \psi_{2}\right) \\
\frac{\Delta b_{C G 2}}{b_{C G 2}}\left(i_{1} * \psi_{1}\right)\end{array}$ & $\begin{array}{l}\frac{\Delta \alpha_{\mathrm{CG} 1}}{\alpha_{\mathrm{CG} 1}}\left(\mathrm{v}_{1} * \phi_{1}\right) \\
\frac{\Delta \alpha_{\mathrm{CG} 2}}{\alpha_{\mathrm{CG} 2}}\left(v_{2} * \phi_{2}\right) .\end{array}$ \\
\hline $\begin{array}{l}\text { Hybrid } \\
\text { gyrator } \\
\text { (HG) }\end{array}$ & $\begin{array}{l}\mathrm{v}_{1}=\mathrm{a}_{\mathrm{HG}} \mathrm{f}_{\mathrm{HG} 1}\left(\mathrm{~b}_{\mathrm{HG}} \mathrm{I}_{2}\right) \\
\mathrm{I}_{1}=\mathrm{a}_{\mathrm{HG}} \mathrm{F}_{\mathrm{HG} 2}\left(\mathrm{~B}_{\mathrm{HG}} \mathrm{v}_{2}\right)\end{array}$ & 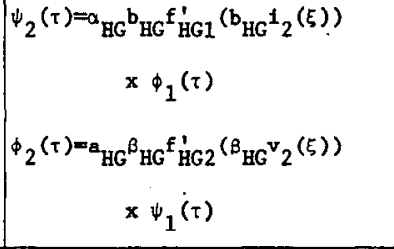 & $\begin{array}{l}\frac{\Delta \mathrm{a}_{\mathrm{HG}}}{\mathrm{a}_{\mathrm{HG}}}\left(i_{1} * \psi_{1}\right) \\
\frac{\Delta \mathrm{b}_{\mathrm{HG}}}{\mathrm{b}_{\mathrm{HG}}}\left(1_{2} * \psi_{2}\right)\end{array}$ & $\begin{array}{l}\frac{\Delta \alpha_{\mathrm{HG}}}{\alpha_{\mathrm{HG}}}\left(\mathrm{v}_{1} * \phi_{1}\right) \\
-\frac{\Delta \beta_{\mathrm{HG}}}{\beta_{\mathrm{HG}}}\left(v_{2} * \phi_{2}\right)\end{array}$ \\
\hline $\begin{array}{l}\text { Impedance } \\
\text { converter } \\
\text { (IC) }\end{array}$ & $\begin{array}{l}v_{1}=a I C^{f} I C 1 \\
\left(\beta_{I C} v_{2}\right) \\
1_{1}=a_{I C} f_{I C 2}\left(b_{I C}{ }^{1}\right)\end{array}$ & 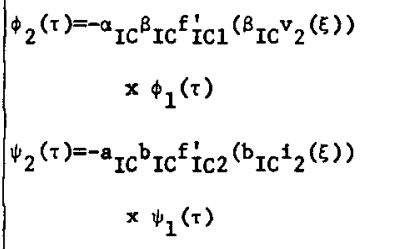 & $\begin{array}{l}-\frac{\Delta \mathrm{a}_{\mathrm{IC}}}{\mathrm{a}_{\mathrm{IC}}}\left(i_{1} * \psi_{1}\right) \\
\frac{\Delta \mathrm{b}_{\mathrm{IC}}}{\mathrm{b}_{\mathrm{IC}}}\left(i_{2} * \psi_{2}\right)\end{array}$ & $\begin{array}{l}\frac{\Delta \alpha_{I C}}{\alpha_{I C}}\left(v_{1} * \phi_{1}\right) \\
-\frac{\Delta B_{I C}}{{ }^{B_{I C}}}\left(v_{2} * \phi_{2}\right)\end{array}$ \\
\hline
\end{tabular}

Similarly, with respect to $\beta_{G}$ of a VCR, we have from Table I and (23)

$$
S_{\beta_{G}}{ }^{v_{2}}=-\left(v_{G} * \phi_{G}\right) .
$$

The contributions due to the parameters of the other one-port nonlinear elements, namely VCC, QCC, FCL, and CCL, may be obtained using Table $I$ in a similar manner. CCT

It may also be seen from Table I and (23) that for a

$$
-S_{a_{C C}{ }^{2}}+S_{b_{C C} v^{2}}=\sum_{e}\left(i_{e} * \psi_{e}\right)
$$

where the summation on the right-hand side of (27) extends over the two ports of the CCT in $N_{1}$ and the corresponding adjoint element in $N_{1}{ }^{A}$. Similarly, we may obtain the sensitivity components for VCT, CVT, VCG, CCG, HG, and IC.

Thus using (23) and Table I we get

$$
-\sum S_{a_{i}}^{{ }^{2}}+\sum S_{b_{j}}^{{ }^{2}}{ }^{2}=\sum_{e}^{\prime}\left(i_{e} * \psi_{e}\right) \text {. }
$$

It should be pointed out that on the left-hand side of (28), only those elements in whose descriptions $a$ and/or $b$ are present are included; further, the prime on the right-hand side indicates that the summation is only over such elements. Thus the VVT is not included. However, for a VVT, the following may be verified directly, using Table I:

$$
\sum_{e}\left(i_{e} * \psi_{e}\right)=0
$$

Hence (28) may be written as

$$
-\sum S_{a_{i}{ }^{2}}+\sum S_{b_{j}}{ }^{v_{2}}=\sum_{e}\left(i_{e} * \psi_{e}\right)
$$

where the right-hand side now includes all the elements of $N_{1}$, even though the sensitivities due to the parameters of a VVT are not included in the left-hand side. Now using (21) in (30), we have

$$
-\sum S_{a_{i}{ }^{22}}+\sum S_{b_{j}}{ }^{{ }^{2}}=\sum_{p .}\left(i_{p} * \dot{\psi}_{p}\right)
$$

The right-hand side of (31) is zero, in view of the excitations and terminations of $N_{1}$ and $N_{1}{ }^{A}$. Hence

$$
\sum S_{a_{i}{ }^{v_{2}}}-\sum S_{b_{j}{ }^{v_{2}}=0 .}
$$

We may also obtain, using (23) and Table I,

$$
\sum S_{\alpha_{i}}{ }^{v_{2}}-\sum S_{\beta_{j}}{ }^{{ }^{2}}=\sum_{e}^{\prime} v_{e} * \psi_{e}
$$

where now the left-hand side includes all the elements whose descriptions contain $\alpha$ and/or $\beta$. Hence the CCT parameters cannot be included on the left-hand side of (33), and thus $\sum$ ? does not contain the CCT elements. However, it again may be verified from Table I that for a $\mathrm{CCT}$

$$
\sum_{e}\left(v_{e} * \phi_{e}\right)=0
$$

Thus

$$
\sum S_{\alpha_{i}}{ }^{v_{2}}-\sum S_{\beta_{j}}^{v_{2}}=\sum_{e}\left(v_{e} * \phi_{e}\right)
$$


where $\sum_{e}$ now includes all the elements of $N_{1}$. By using (20) in (35), we get

$$
\sum S_{\alpha_{i}{ }^{v_{2}}}-\sum S_{\beta_{j}{ }^{{ }_{2}}}=\sum_{p}\left(v_{p} * \phi_{p}\right) .
$$

In view of terminations and excitations in $N_{1}$ and $N_{1} \boldsymbol{A}$, we have

$$
\sum_{p}\left(v_{p} * \phi_{p}\right)=\left(v_{1} * \phi_{1}\right)+v_{2}
$$

which need not be an invariant, in general. Hence we have no invariance, in general, for the expression

$$
\sum S_{\alpha_{i}{ }^{{ }^{2}}}-\sum S_{\beta_{j}{ }^{{ }^{2}} \text {. }}
$$

\section{Case 2: Current Response Due to Voltage Excitation}

Let $i_{2}$ denote the short-circuit current response at port 2 of $N_{1}$ due to voltage excitation $v_{1}$ at port 1 , all other ports being short circuited. Let port 2 of $N_{1}{ }^{A}$ be excited by an impulse voltage function $\delta(\tau)$, all other ports being short circuited; then the left-hand side of (2) can be written as

$$
-\int_{0}^{t} \Delta i_{2}(\xi) \delta(t-\xi) d \xi=-\Delta i_{2}(t)
$$

Following the same procedure as used in Case 1, we have, from the right-hand side of (2), (39), and Table I,

$$
\sum S_{a_{i}}{ }^{{ }^{2}}-\sum S_{b_{j}}{ }^{{ }_{2}}=\sum_{e}\left(i_{e} * \psi_{e}\right)=\sum_{p}\left(i_{p} * \psi_{p}\right) .
$$

But in view of the excitations and terminations in $N_{1}$ and $N_{1}{ }^{A}$, we have

$$
\sum_{p}\left(i_{p} * \psi_{p}\right)=i_{2}
$$

Thus from (40) and (41) we get

$$
\sum S_{a_{i}}{ }^{i_{2}}-\sum S_{b_{j}}^{i_{2}}=i_{2} \text {. }
$$

\section{Case 3: Voltage Response Due to Current Excitation}

Let us now consider the open-circuit voltage response $v_{2}$ at port 2 due to a current excitation $i_{1}$ at port 1 of the network $N_{2}$, all other ports being open. Let the second port of $N_{2}{ }^{A}$, the adjoint of $N_{2}$, be excited by an impulse current function $\delta(\tau)$, all other ports of $N_{2}{ }^{A}$ being open. Then the left-hand side of (2) can be written as

$$
\int_{0}^{t} \Delta v_{2}(\xi) \delta(t-\xi) d \xi=\Delta v_{2}(t)
$$

Following the same procedure as in Case 1, and using the right-hand side of (2), (43), and Table I, we can show that

$$
\sum S_{\alpha_{i}}{ }^{v_{2}}-\sum S_{\beta_{j}{ }^{v_{2}}}=\sum_{e}\left(v_{e} * \phi_{e}\right) .
$$

Again using (21), we have

$$
\sum_{e}\left(v_{e} * \phi_{e}\right)=\sum_{p}\left(v_{p} * \phi_{p}\right)=v_{2}
$$

in view of the excitations and terminations in $N_{2}$ and $\mathrm{N}_{2}{ }^{A}$. Hence (44) reduces to

$$
\sum S_{\alpha_{i}{ }^{2}}-\sum S_{\beta_{j}{ }^{2}}=v_{2} .
$$

\section{Case 4: Current Response Due to Current Excitation}

Let us consider the short-circuit current response $i_{2}$ at port 2 due to a current excitation $i_{1}$ at port 1 of $N_{2}$, all other ports being short circuited. Let port 2 of $N_{2}{ }^{A}$ be excited by an impulse voltage function $\delta(\tau)$, with port 1 open and all other ports short circuited. Then it follows that the left-hand side of (2) becomes

$$
-\int_{0}^{t} \Delta i_{2}(\xi) \delta(t-\xi) d \xi=-\Delta i_{2}(t) .
$$

Following the same procedure as in Case 1, and using the right-hand side of (2), (46), Table I, and the excitation as well as the terminating conditions in $\mathrm{N}_{2}$ and $\mathrm{N}_{2}{ }^{A}$, we can show that

$$
\sum S_{\alpha_{i}}{ }^{i_{2}}-\sum S_{\beta_{j}}{ }^{i_{2}}=0 .
$$

We shall now establish similar sensitivity-invariant relations with different types of excitations and responses when the change in the response due to a change in the amplitude of excitation is also considered.

\section{Case A : Voltage Response Due to Voltage Excitation}

Let us consider the open-circuit voltage response $v_{2}$ at port 2.due to a voltage excitation $v_{1}$ at port 1 of $N_{2}$, all other ports being open circuited. Let the second port of $N_{2}{ }^{A}$ be excited by an impulse current function $\delta(\tau)$, with port 1 shorted and all other ports open.

Let us first consider the variation in the response due to a variation in the amplitude of the excitation only. Since the variation in the response due to the parameters $a^{\prime}$ s, $b$ 's, $\alpha$ 's, and $\beta$ 's of the network elements have been assumed to be zero, (2) can be written as

$$
\int_{0}^{t} \Delta v_{1}(\xi) \phi_{1}(t-\xi) d \xi+\Delta v_{2}(t)=0 .
$$

Let

$$
v_{1}=V_{1} f(t) .
$$

Then the variation in $v_{1}$ due to a change in the amplitude $V_{1}$ of $v_{1}$ is

$$
\Delta v_{1}=\Delta_{V 1} f(t) .
$$

Hence (48) may be written as

$$
\Delta v_{2}=-\left(\Delta V_{1} / V_{1}\right)\left(v_{1} * \phi_{1}\right) .
$$

Thus

$$
S_{V_{1}}^{v_{2}}=-\left(v_{1} * \phi_{1}\right) .
$$

We also have, from (21), in view of the terminating and excitation conditions in $N_{2}$ and $N_{2}{ }^{A}$,

$$
\left(v_{1} * \phi_{1}\right)+v_{2}=\sum_{e}\left(v_{e} * \phi_{e}\right) .
$$


Thus from (50) and (51) we get

$$
-S_{V_{1}}^{v^{2}}+v_{2}=\sum_{e}\left(v_{e} * \phi_{e}\right)
$$

We already have shown in Case 1 that

$$
\sum S_{\alpha_{i}}^{v^{2}}-\sum S_{\beta_{j}}{ }^{2}=\sum_{e}\left(v_{e} * \phi_{e}\right)
$$

Hence from (52) and (35) we have

$$
S_{V_{1}}^{{ }^{2}}+\sum S_{\alpha_{i}}{ }^{{ }^{2}}+\sum S_{\beta_{j}}^{{ }^{2}}=v_{2} \text {. }
$$

\section{Case B : Voltage Response Due to Current Excitation}

Consider the open-circuit voltage response $v_{2}$ at port 2 due to current excitation $i_{1}$ at port 1 of $N_{1}$. If there is a small variation in the amplitude $I_{1}$ of the excitation $i_{1}=I_{1} f(t)$, then it can be shown, following the same procedure as used in Case $A$, that

$$
S_{I_{1}}^{v_{2}}+\sum S_{a_{i}{ }^{2}}-\sum S_{b_{j}}^{v_{2}}=0
$$

\section{Case C: Current Response Due to Voltage Excitation}

Consider the short-circuit current response $i_{2}$ at port 2 due to voltage excitation $v_{1}$ at port 1 of network $N_{2}$. If there is a small change in the amplitude $V_{1}$ of excitation $v_{1}=V_{1} f(t)$, then it readily can be shown that

$$
S_{V_{1}}^{{ }^{i 2}}+\sum S_{\alpha_{i}}^{{ }^{i 2}}-\sum S_{\beta_{j}}{ }^{{ }^{2}}=0
$$

\section{Case D: Current Response Due to Current Excitation}

Consider a short-circuit current response $i_{2}$ at port 2 due to current excitation $i_{1}$ at port 1 of $N_{1}$. If there is a slight variation in the amplitude $I_{1}$ of excitation $i_{1}=I_{1} f(\iota)$, then it readily can be shown that

$$
S_{I_{1}}^{i_{2}}+\sum S_{a_{i}}^{{ }^{i_{2}}}-\sum S_{b_{j}}{ }^{i_{2}}=i_{2} \text {. }
$$

The different sensitivity-invariant relationships derived above for networks $N_{1}$ and $N_{2}$ are summarized below.

1) For network $N_{1}$ :

a) For voltage excitation

$$
\begin{aligned}
& \sum S_{a_{i}{ }^{{ }_{2}}}-\sum S_{b_{j}{ }^{{ }_{2}}}=0 \\
& \sum S_{a_{i}{ }^{i_{2}}}-\sum S_{b_{j}}{ }^{{ }^{i_{2}}}=i_{2} .
\end{aligned}
$$

b) For current excitation $i_{1}=I_{1} f(t)$

$$
\begin{aligned}
& S_{I_{1}{ }^{v_{2}}}+\sum S_{a_{i}{ }^{v_{2}}-\sum S_{b_{j}}{ }^{v_{2}}=0}=0 \\
& S_{I_{1}}{ }^{i_{2}}+\sum S_{a_{i}}{ }^{i_{2}}-\sum S_{b_{j}}{ }^{i_{2}}=i_{2} .
\end{aligned}
$$

2) For network $N_{2}$ :

a) For voltage excitation $v_{1}=V_{1} f(t)$

$$
\begin{aligned}
& S_{V_{1}}{ }^{v_{2}}+\sum S_{\alpha_{i}}{ }^{{ }^{2}}-\sum S_{\beta_{j}}{ }^{i_{2}}=v_{2} \\
& S_{V_{1}{ }^{i_{2}}}+\sum S_{\alpha_{i}{ }^{i 2}}-\sum S_{\beta_{j}}{ }^{i_{2}}=0 \text {. }
\end{aligned}
$$

b) For current excitation

$$
\begin{aligned}
& \sum S_{\alpha_{i}{ }^{{ }^{2}}}-\sum S_{\beta_{j}}{ }^{{ }^{2}}=0 \\
& \sum S_{\alpha_{i}}{ }^{{ }^{2}}-\sum S_{\beta_{j}}{ }^{{ }^{2} 2}=v_{2} .
\end{aligned}
$$

It should be pointed that, if in the network $N$ all the initial conditions for VCC's, QCC's, CCL's, and FCL's are $z$ ero, that is,

$$
V_{C}(0)=q_{C}^{\prime}(0)=i_{L}(0)=\eta_{L}(0)=0
$$

then the two classes $N_{1}$ and $N_{2}$ are indistinguishable. In such a case, all the relations (57)-(64) apply.

\section{EXAMPLE}

Consider the circuit given in Fig. 1, where the diode is described by

$$
i_{1}=a\left(e^{\beta v_{2}}-1\right) .
$$

Let $V_{1}$ be a dc excitation and let $v_{2}$ be the response. Since the nctwork may be considered as belonging to either class $N_{1}$ or $N_{2}$, relation (57), as well as (61), applies.

The linear resistors may be considered either as VCR's,

$$
\begin{aligned}
& i=G_{1} v=G_{1}\left(V_{1}-v_{2}\right) \\
& i_{2}=G_{2} v_{2}
\end{aligned}
$$

or as CCR's,

$$
\begin{aligned}
& v=\left(V_{1}-v_{2}\right)=R_{1} i \\
& v_{2}=R_{2} i_{2} .
\end{aligned}
$$

First let us consider the linear resistors as VCR's. Then we may identify $G_{1}$ and $G_{2}$ as $a_{1}$ and $a_{2}$ or $\beta_{1}$ and $\beta_{2}$. In order to use (57), $G_{1}$ and $G_{2}$ will have to be identified with $a_{1}$ and $a_{2}$ and not with $\beta_{1}$ and $\beta_{2}$, since otherwise the left-hand side of $(57)$ will not include the resistors (but at the same time, the summation is over all the elements of the network). Also, in order to use (61), $G_{1}$ and $G_{2}$ will have to be identified with $\beta_{1}$ and $\beta_{2}$ and not with $a_{1}$ and $a_{2}$, since otherwise the left-hand side of (61) will not include the linear resistors.

Thus from (57) we get

$$
S_{G_{1}}^{{ }^{2}}+S_{G_{2}}^{v_{2}}+S_{a}{ }^{v 2}=0
$$

while from (61) we have

$$
S_{V_{1}}^{v_{2}}-S_{G_{1}{ }^{v_{2}}}-S_{G_{2}{ }^{v_{2}}}-S_{\beta}^{v_{2}}=v_{2} \text {. }
$$

If we now consider the linear resistors as CCR's, then we may identify $R_{1}$ and $R_{2}$ with $b_{1}$ and $b_{2}$ or $\alpha_{1}$ and $\alpha_{2}$. For reasons similar to the one given above, $R_{1}$ and $R_{2}$ have to be identified with $b_{1}$ and $b_{2}$ in (57), and with $\alpha_{1}$ and $\alpha_{2}$ in (61). Then we get

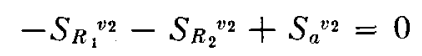

$$
\begin{aligned}
& S_{V_{1}}^{v_{2}}+S_{R_{1}{ }^{v_{2}}}+S_{R_{2}}^{v_{2}}-S_{\beta}^{v_{2}}=0 \text {. }
\end{aligned}
$$

Of course, (67) and (65), as well as (68) and (66), are the same.

Now if $i_{2}$ is the response, then (58) and (62) apply. Whether we consider the linear resistors to be VCR's or CCR's, they give rise to

$$
\begin{aligned}
S_{G_{1}}{ }^{i_{2}}+S_{G_{2}}{ }^{i_{2}}+S_{a}{ }^{i_{2}} & =i_{2} \\
S_{V_{1}}{ }^{i_{2}}-S_{G_{1}}{ }^{i_{2}}-S_{G_{2}}{ }^{i_{2}}-S_{\beta}{ }^{i_{2}} & =0 .
\end{aligned}
$$




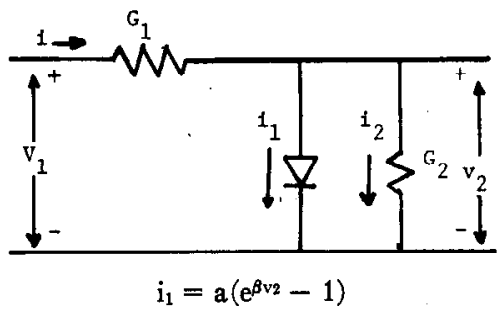

Fig. 1. Diagram for the example.

It may be verified directly that (65) holds by writing Kirchhoff's equation for the network as

$$
G_{1} V_{1}=G_{1} v_{2}+a\left(e^{\beta v_{2}}-1\right)+G_{2} v_{2}
$$

then differentiating (71) with respect to $G_{1}, G_{2}$, and $a$, adding to obtain

$$
\left(G_{1}+G_{2}+a \beta e^{\beta v_{2}}\right)\left(S_{G_{1}{ }^{2}}+S_{G_{2}}^{v_{2}}+S_{a}^{v_{2}}\right)=0
$$

and finally by observing that $G_{1}+G_{2}+a \beta e^{\beta p 2} \neq 0$. In a similar fashion, (68), (69), and (70) may be verified.

\section{Conclusions}

In this paper some sensitivity-invariant relationships have been established for certain classes of nonlinear networks with different types of excitations and responses.

We note that higher order sensitivity invariants for networks $N_{1}$ and $N_{2}$ may be established using the following relation proved in [10]:

$$
X_{k+1}=\sum_{i=1}^{n} p_{i} \frac{\partial X_{k}}{\partial p_{i}}-k X_{k}
$$

where $X_{j}$ denotes the sum of $j$ th order sensitivities over the set of parameters $\left(p_{i}, i=1,2, \cdots, n\right)$.
Further, if the quadratic sensitivity index $\phi$ is defined for nonlinear networks in the same manner as it is done for linear networks [11], then the sensitivity-invariant relationships established in this paper can be used to obtain lower bounds for $\phi$, following the approach used in [6], [10], and [12].

\section{ACKNowledgment}

The authors would like to thank Dr. V. Ramachandran for some helpful discussions.

\section{REFERENCES}

[1] M. L. Blostein, "Sensitivity analysis of parasitic effects in resistance-terminated LC two-ports," IEEE Trans. Circuit Theory, vol. CT-14, pp. 21-25, Mar. 1967.

[2] _ "Some bounds on sensitivity in RLC networks," in Proc. 1st Allerton Conf., pp. 488-501, 1963.

[3] A. G. J. Holt and J. K. Fidler, "Summed sensitivity of network functions," Electrm. Lett., pp. 85-87, Mar. 1968.

[4] A. G. J. Holt and M. R. Lee, "Summed sensitivity of active RC networks," Electron. Lett., pp. 385-386, June 1968.

[5] E. S. Kuh and C. G. Lau, "Sensitivity invariants of continuously equivalent networks," IEEE Trans. Circuit Theory, vol. CT-15, pp. 175-177, Sept. 1968.

[6] C. Schmidt and R. Kasper, "On minimum sensitivity networks," IEEE Trans. Circuit Theory, vol. CT-14, pp. 438-440, Dec. 1967.

[7] B. J. Leon and C. F. Yokomoto, "Generation of a class of equivalent networks and its sensitivities," IEEE Trans. Circuit Theory, vol. CT-19, pp. 2-8, Jan. 1972

[8] M. Sablatash and R. Seviora, "Sensitivity invariants for scattering matrices," IEEE Trans. Circuit Theory (Corresp.), vol. CT. 18, pp. 282-284, Mar. 1971 .

[9] S. W. Director and R. A. Rohrer, "The generalized adjoint network and network sensitivities," IEEE Trans. Circuit Theory, vol. CT-16, pp. 318-323, Aug. 1969.

[10] M. N. S. Swamy, C. Bhushan, and K. Thulsiraman, "Sensitivity invariants for active lumped/distributed networks," in Pror. 1972 IEEE Int. Symp. Circuit Theory, pp. 16-20.

11] ]. D. Schoeffler, "The synthesis of minimum sensitivity networks," IEEE Trans. Circuit Theory, vol. CT-14, pp. 271-276, June 1964.

[12] M. N. S. Swamy, C. Bhushan, and K. Thulasiraman, "Bounds on the sum of element sensitivity magnitudes for network functions," in Proc. 1972 IEEE Int. Symp. Circuit Theory, pp. 21-25.

[13] S. R. Parker, "Sensitivity: Old questions, some new answers," IEEE Trans. Circuit Theory, vol. CT-18, pp. 27-35, Jan. 1971.

[14] L. O. Chua, Introduction to Nonlinear Network Theory. New York: McGraw-Hill, 1969.

[15] A. K. Seth and K. Singhal, "Time domain sensitivity using the adjoint network," Electron. Lett., pp. 563-565, Sept. 1971. 